\title{
Alpha Phase Determines Successful Lexical Decision in Noise
}

\author{
๑Antje Strauß, ${ }^{1}$ Molly J. Henry, ${ }^{1}{ }^{\circledR}$ Mathias Scharinger, ${ }^{1}$ and $\odot$ Jonas Obleser ${ }^{1,2}$ \\ ${ }^{1}$ Max Planck Research Group “Auditory Cognition," Max Planck Institute for Human Cognitive and Brain Sciences, 04103 Leipzig, Germany, and \\ ${ }^{2}$ Department of Psychology, University of Lübeck, 23562 Lübeck, Germany
}

\begin{abstract}
Psychophysical target detection has been shown to be modulated by slow oscillatory brain phase. However, thus far, only low-level sensory stimuli have been used as targets. The current human electroencephalography (EEG) study examined the influence of neural oscillatory phase on a lexical-decision task performed for stimuli embedded in noise. Neural phase angles were compared for correct versus incorrect lexical decisions using a phase bifurcation index (BI), which quantifies differences in mean phase angles and phase concentrations between correct and incorrect trials. Neural phase angles in the alpha frequency range $(8-12 \mathrm{~Hz})$ over right anterior sensors were approximately antiphase in a prestimulus time window, and thus successfully distinguished between correct and incorrect lexical decisions. Moreover, alpha-band oscillations were again approximately antiphase across participants for correct versus incorrect trials during a later peristimulus time window $(\sim 500 \mathrm{~ms})$ at left-central electrodes. Strikingly, lexical decision accuracy was not predicted by either event-related potentials (ERPs) or oscillatory power measures. We suggest that correct lexical decisions depend both on successful sensory processing, which is made possible by the alignment of stimulus onset with an optimal alpha phase, as well as integration and weighting of decisional information, which is coupled to alpha phase immediately following the critical manipulation that differentiated words from pseudowords. The current study constitutes a first step toward characterizing the role of dynamic oscillatory brain states for higher cognitive functions, such as spoken word recognition.
\end{abstract}

Key words: bifurcation index; decision weighting; EEG; lexical decision; neural oscillations; sensory selection

\section{Introduction}

Human psychophysical performance for detection and discrimination of low-level stimuli has been found to depend on slow prestimulus oscillatory brain states across domains (visual: Varela et al., 1981; Hanslmayr et al., 2007; van Dijk et al., 2008; Busch et al., 2009; Schubert et al., 2009; Cravo et al., 2013; Spaak et al., 2014; auditory: Lakatos et al., 2005; Henry and Obleser, 2012; audiovisual: Keil et al., 2014). These findings relate neural phase to neural excitability fluctuations, such that performance is best for targets coinciding with the excitable phase of a neural oscillation, and worst for targets coinciding with the inhibitory phase. Going beyond low-level perception, we ask here whether higher cognitive functions, such as speech processing, would also depend on neural phase. Although recently proposed models would predict a dependence of speech processing on neural oscillatory phase (Ghitza, 2011; Gagnepain et al., 2012; Giraud and Poeppel, 2012), no experimental evidence has been gathered so far.

\footnotetext{
Received Aug. 12, 2014; revised Jan. 13, 2015; accepted Jan. 15, 2015

Author contributions: A.S., M.S., and J.0. designed research; A.S. performed research; M.J.H. and J.0. contributed unpublished reagents/analytic tools; A.S. and M.J.H. analyzed data; A.S., M.J.H., M.S., and J.O. wrote the paper.

This work was supported by the Max Planck Society to A.S., M.J.H., M.S., and J.0., and a Max Planck Research Group grant to J.0. We thank two anonymous reviewers wo helped improve the paper, and Dunja Kunke who helped record the data.

The authors declare no competing financial interests.

Correspondence should be addressed to either Antje Strauß or Jonas Obleser, Max Planck Research Group “Auditory Cognition," Max Planck Institute for Human Cognitive and Brain Sciences, Stephanstraße 1A, 04103 Leipzig, Germany.E-mail: strauss@cbs.mpg.de or obleser@cbs.mpg.de.

DOI:10.1523/JNEUROSCI.3357-14.2015

Copyright $\odot 2015$ the authors $\quad 0270-6474 / 15 / 353256-07 \$ 15.00 / 0$
}

One elegant task that can bridge psychophysical aspects of performance (detection or discrimination) with speech processing is the auditory lexical decision task (Marslen-Wilson, 1980): listeners are presented with words, as well as word-like stimuli (i.e., pseudowords), and have to judge whether they heard a meaningful word or not. Parallel to low-level discrimination studies, we made the lexical decision task "near-threshold" by embedding speech in individually titrated levels of white noise, which increased the difficulty of the task and, purposefully, the amount of errors. We simultaneously recorded the electroencephalogram and hypothesized that a dependence of lexicaldecision accuracy on low-frequency neural oscillatory phase should be observed.

Here, we were interested in the role of alpha $(8-12 \mathrm{~Hz})$ and theta $(3-7 \mathrm{~Hz})$ neural phase for lexical decision performance. Instantaneous alpha phase has previously been linked to lowlevel detection and discrimination performance not only in the visual (Mathewson et al., 2009; Busch and VanRullen, 2010; Romei et al., 2010), but also in the auditory domain (Rice and Hagstrom, 1989; Neuling et al., 2012). Critically, alpha phase has been found to modulate neuronal firing and to determine the neural phase associated with best discrimination performance (Haegens et al., 2011). Discrimination performance in lexical decision may also depend on syllabic processing and thus potentially be indexed by oscillatory activity in the theta range $(\sim 4 \mathrm{~Hz})$ with oscillation periods corresponding to the average syllable duration of $250 \mathrm{~ms}$ ( $\mathrm{Ng}$ et al., 2012; Peelle and Davis, 2012; Gross et al., 2013; Doelling et al., 2014; note that also Busch et al., 2009 reported a prestimulus phase bifurcation effect in the $7 \mathrm{~Hz}$ range). Similar to alpha, theta oscillations have been linked to 
A Trial design
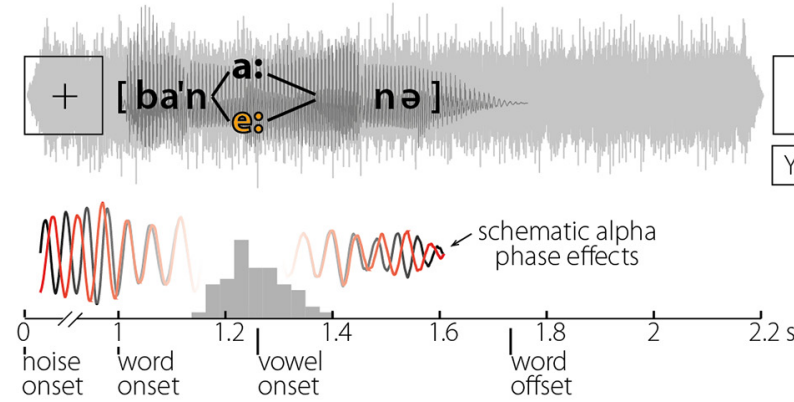

B Analysis scheme
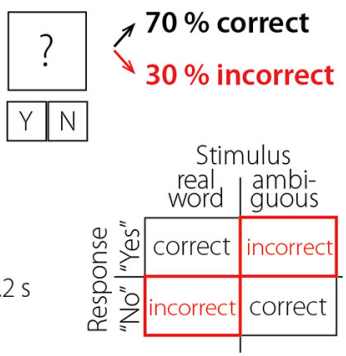

C Performance

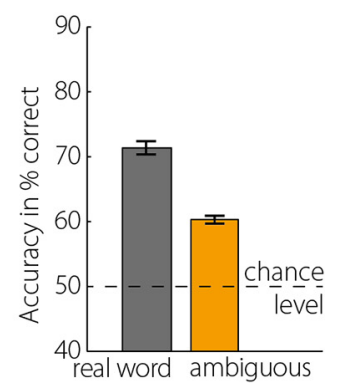

Figure 1. Trial design and behavioral measures. $A$, Trial design. Lexical stimuli were presented against a white-noise background. A histogram of the critical vowel onsets is shown schematically in relation to the timing of the two alpha phase effects below the example trial. Average word length was $0.74 \pm 0.08 \mathrm{~s}$ (M \pm 1 SD). Delayed lexical decision was prompted by a question mark. $\boldsymbol{B}$, Analysis scheme. Seventy percent correct was targeted with individual SNRs. For the analysis, correct trials comprised trials on which participants responded Yes to a real word or No to the ambiguous counterpart as illustrated by the cross-tabulation. C, Behavioral results. Participants performed better for real words than for ambiguous pseudowords. However, performance for both stimulus types was significantly above chance.

neuronal firing (Kayser et al., 2012) and can impact auditory detection performance ( $\mathrm{Ng}$ et al., 2013).

Our data show that the accuracy of auditory lexical decision depends on the instantaneous phase of alpha oscillations: Stimuli that were judged incorrectly fell into an alpha phase opposite to that for stimuli that were later judged correctly in a prestimulus time window, as well as in a second, peristimulus time window.

\section{Materials and Methods}

Participants

Eleven participants ( $25.1 \pm 1.6$ years, $\mathrm{M} \pm \mathrm{SD} ; 7$ females) gave informed consent to take part in the experiment. All were native speakers of German, right-handed, with self-reported normal hearing abilities, and no history of neurological or language-related problems. They received financial compensation for their participation. All procedures had ethical approval from the Ethics Committee of the University of Leipzig.

\section{Stimulus material}

Stimuli were real words and their pseudoword counterparts (Raettig and Kotz, 2008; Strauß et al., 2014b). Pseudowords were created as follows: From a list of 60 tri-syllabic concrete German nouns ('real' words, e.g., /banane/, [engl. banana]) two types of pseudowords were derived, 'ambiguous' pseudowords, by exchanging the nucleus vowels of the second syllables across words (e.g., /banene/), and 'opaque' pseudowords by scrambling the syllables across words while keeping the position-in-word fixed (e.g., /bapossner/). Furthermore, 60 'abstract' real words (e.g., /botanik/, [engl. botany]) served as fillers to ensure a balanced word-pseudoword ratio and were not analyzed further. In sum, the experimental corpus consisted of 240 lexical stimuli with a mean length of $754.2 \pm 83.5$ $\mathrm{ms}(\mathrm{M} \pm \mathrm{SD})$. For the opaque pseudowords, accuracy was very high ( $89 \pm 8 \%$ correct, $\mathrm{M} \pm \mathrm{SD}$ ), thus there were not enough incorrect trials to allow for a direct comparison of correct and incorrect trials. Therefore in the following, we focused on the noise-induced vowel confusion between real words and ambiguous pseudowords that lead to decisions about whether an item was a word or not.

All words and pseudowords were spoken by a trained female speaker and digitized at $44.1 \mathrm{kHz}$. Postprocessing included down-sampling to $22.05 \mathrm{kHz}$, cutting at zero crossings closest to articulation on- and offsets, and root mean square (RMS) normalization.

\section{Materials and Methods}

Before each experimental EEG session, individual signal-to-noise ratios (SNR) were determined by means of an adaptive tracking procedure. During adaptive tracking, participants were presented with the second syllables extracted from the real words and their ambiguous-pseudoword counterparts. On each trial, the participant heard two successive syllables embedded in white noise and indicated whether the vowels in each pair were "same" or "different." Intensity of the syllables relative to the white noise was adjusted according to a two-down-one-up staircase procedure that estimated the signal-to-noise ratio (SNR) targeting 70.7\% accuracy (Levitt, 1971). Resulting average SNR was $-10.22 \pm 1.95 \mathrm{~dB}(\mathrm{M} \pm \mathrm{SD})$. That way, the identical white noise was used for all participants in each run of the adaptive tracking procedure, as well as in all trials of the following EEG experiment.

Next, a short familiarization for the trial timing was provided during which participants made lexical decisions in noise $\sim 10$ additional items from Raettig and Kotz (2008) that were not used in the present experiment. During the EEG experiment, participants heard words and pseudowords embedded in white noise and indicated via button press whether they heard a real word or not ("Yes"/"No"). Button order (left/ right for Yes/ No responses) was counterbalanced across participants. On each trial, the white noise started $1 \mathrm{~s}$ before (pseudo)word onset, coincident with the appearance of a fixation cross, and lasted for $2.2 \mathrm{~s}$ in total (Fig. 1A). After $2.2 \mathrm{~s}$, the fixation cross-changed to a question mark that prompted the lexical decision response. Trial timing was chosen based on a previous study in our lab using the same paradigm without noise (Strauß et al., 2014b) and allowed artifact-free estimations of time-frequency representations (see Data analysis).

Each participant listened to 240 stimuli ( 120 words, 120 pseudowords) in an individually pseudorandomized sequence. That is, each participant heard both the "real" and the "ambiguous" versions of each word. The order of occurrence for a given real word and its pseudoword counterpart was counterbalanced across participants to control for potential interfering effects of previous exposure to the respective complementary item. For the same reason, the distance between a word and its pseudoword counterpart was maximized (i.e., on average 120 other items inbetween). Listeners paused after each block of 60 trials. Overall duration of the experimental procedure was $\sim 30 \mathrm{~min}$.

\section{Data acquisition and preprocessing}

The electroencephalogram (EEG) was recorded from $64 \mathrm{Ag}-\mathrm{AgCl}$ electrodes positioned according to the extended 10-20 standard system on an elastic cap with a ground electrode mounted on the sternum. Bipolar horizontal and vertical electrooculograms were recorded for ocular artifact-rejection purposes. All impedances were kept at $<5 \mathrm{kOhm}$. Signals were referenced online against the left mastoid, and digitized with a sampling rate of $500 \mathrm{~Hz}$ and a passband of DC to $140 \mathrm{~Hz}$. Individual electrode positions were determined after EEG recording with the Polhemus FASTRAK electromagnetic motion tracker.

EEG preprocessing was done off-line using the open-source Fieldtrip toolbox (Oostenveld et al., 2011) for MATLAB (MathWorks). To avoid edge effects at low frequencies, broad epochs were defined ranging between $-700 \mathrm{~ms}$ [excluding event-related potentials (ERPs) due to noise onset] and $2100 \mathrm{~ms}$ relative to (pseudo)word onset. Data were bandpass filtered from 0.1 to $100 \mathrm{~Hz}$ using an acausal fourth-order two-pass Butterworth filter (using the MATLAB-inbuilt filtfilt function, thus effectively doubling the filter order), and for ERP analysis only, re-referenced to combined mastoids (time-frequency analyses used re-referencing to 
average reference, see Data analyses, below). To reject systematic artifacts, independent component analysis (ICA) was applied and components comprising eye movement, heartbeat, and muscle artifacts were rejected according to definitions provided by Debener et al. (2010). After ICA, an automatic artifact-rejection routine removed single trials for which within-channel peak-to-peak range exceeded $120 \mu \mathrm{V}$. On average, $2.7 \pm 3.0(\mathrm{M} \pm \mathrm{SD})$ trials were rejected per participant. The resulting clean data were used for subsequent data analyses.

\section{Data analyses}

Phase analysis. Time-frequency representations (TFRs) were estimated from single-trial data so that we could assess the effects of phase and power on lexical decisions. Epoched, filtered, artifact-rejected timedomain data were re-referenced to average reference (Strauß et al., 2014b). Subsequently, Morlet wavelets were applied to single-trial TFRs in $20 \mathrm{~ms}$ steps with a frequency-specific window width to account for the tradeoff between higher-frequency resolution for lower frequencies and higher time resolution for higher frequencies. Therefore, TFRs for logarithmically spaced frequencies from 3 to $30 \mathrm{~Hz}$ were convolved with linearly increasing window widths ranging from 2 to 12 cycles. Phase and power values were then estimated at each channel $\times$ frequency $\times$ time point from the complex output of the wavelet convolution.

For the analysis of phase data, we calculated a phase bifurcation index (BI), $\varphi$, suggested by Busch et al. (2009). First, trials were split based on accuracy (i.e., correct vs incorrect responses) for each participant. Then, we calculated intertrial phase coherence (ITPC; $0 \leq$ ITPC $\geq 1$ ) separately for correct trials, for incorrect trials, and for all trials taken together. Last, to compute the phase bifurcation index, $\varphi$, ITPC for correct, incorrect, and all trials were combined according to the following formula:

$$
\varphi=\left(\mathrm{ITPC}_{\text {correct }}-\mathrm{ITPC}_{\text {all }}\right) \times\left(\mathrm{ITPC}_{\text {incorrect }}-\mathrm{ITPC}_{\text {all }}\right)
$$

BIs were calculated separately for each channel $\times$ frequency $\times$ time bin. A positive $\mathrm{BI}$ indicates that two conditions have similar intertrial phase coherence values but differ in their mean phases ( $>\pi / 2$ radians). A negative $\mathrm{BI}$, by contrast, indicates that one condition is more phase-locked than the other; i.e., angles of one condition are randomly distributed, whereas angles of the other condition concentrate toward a certain direction. Further details on the BI can be found in the study by Busch et al. (2009).

As expected, the number of trials was not balanced between correct (number of trials per subject $=75.36 \pm 8.87$ ) and incorrect trials (number of trials per subject $=39 \pm 8.28$; Fig. $1 B$, see analysis scheme). To account for this inequality, which can bias estimates of ITPC (Lachaux et al., 1999; Ding and Simon, 2013), we performed a randomization test analogous to the Monte Carlo method described by Maris and Oostenveld (2007) to obtain a robust measure of the BI in each participant. For each participant, the number of trials to be selected was equal to $75 \%$ of the amount of incorrect trials (the category with the smallest number of trials) resulting in $29.45 \pm 6.25$ trials per condition. On each of 1000 iterations, trials were randomly selected without replacement from the set of correct and incorrect trials. From ITPC estimates for correct, incorrect, and all selected trials, a single BI was calculated. The mean bifurcation index over these 1000 repetitions was used per participant for further statistical analyses.

On the group level, we tested BIs against zero separately for the alpha $(8-12 \mathrm{~Hz})$ and the theta $(3-5 \mathrm{~Hz})$ frequency bands for each time point in the range between -0.35 and $1.1 \mathrm{~s}$ with respect to (pseudo)word onset using the Monte Carlo randomization method (1000 repetitions) with cluster correction as implemented in FieldTrip. The time window was chosen such that edge effects of TFR estimation for lowest frequencies were avoided and stimulus offset responses at $1.2 \mathrm{~s}$ post-(pseudo)word onset (i.e., the end of the masking noise) were excluded.

Further analyses. To further characterize the phase effects found via the test of the $\mathrm{BI}$ and to test for potential confounds, we also evaluated alpha and theta ITPC, absolute alpha and theta power, and ERPs. For the ITPC analysis, the differences between ITPC $\mathrm{C}_{\text {correct }}$ and $\mathrm{ITPC}_{\text {incorrect }}$ trials $(8-12 \mathrm{~Hz}$ and $3-5 \mathrm{~Hz}$; from -0.35 to $1.1 \mathrm{~s})$ as estimated for the BI calculation (i.e., 1000 iterations) were averaged per participant and submitted to a two-tailed single-sample $t$ test against zero with cluster correction using the Monte Carlo randomization method (1000 repetitions).
For power estimates, we squared the magnitude (complex modulus) of single-trial Fourier data. Analogous to the phase analysis, the same amount of trials for correct and incorrect trials were selected as described in the previous paragraph. Subsequently, their power difference was calculated, and the mean $>1000$ of such differences was taken per subject. The group-level analysis on power was the same as described in the previous paragraph for BI and ITPC analyses.

For analysis of ERPs, the epoched, filtered, and artifact-rejected timedomain data were filtered with an acausal sixth-order two-pass Butterworth low-pass filter at $15 \mathrm{~Hz}$. For baseline correction, a time window from -200 to $0 \mathrm{~ms}$ pre-(pseudo)word onset (i.e., during the masking noise) was chosen. Amplitudes were then averaged in selected time windows over selected channels. Time window and channel selection were based on the peristimulus BI cluster. A pairwise $t$ test compared ERP amplitudes of correct versus incorrect trials across subjects.

Effect sizes. For simple $t$ statistics (dependent and independent samples $t$ tests), we estimated the effect size measure $r_{\text {equivalent }}$, here denoted $r$, which is bound between 0 and 1 (Rosenthal, 1994; Rosenthal and Rubin, 2003). Effect sizes for multiple $t$ tests (e.g., for all channel $\times$ frequency $\times$ time bins belonging to a significant cluster) were estimated by averaging $r$ values across individual tests constituting the cluster (denoted $R$ ).

\section{Results}

\section{Accuracy of lexical decisions}

As shown in Figure 1C, participants achieved an average accuracy near that targeted by the adaptive tracking procedure for real words $(71.4 \pm 1.02 \%)$. Although slightly worse, accuracy for the ambiguous pseudowords was still better than chance $(60.3 \pm$ $0.61 \%$; $t$ test against 50\%: $\left.p=0.0005, t_{(10)}=5.1, r=0.85\right)$.

\section{Neural phase in the alpha band predicts lexical-decision accuracy}

To evaluate differences in neural phase, the bifurcation index (BI) suggested by Busch et al. (2009) was calculated for each channel-time-frequency bin. A positive BI indicates that two conditions exhibit similar phase concentration but differ in their mean phases ( $>\pi / 2$ radians). Nonparametric permutation tests of the BI against zero revealed two positive clusters (i.e., similar phase concentration but opposite mean phases for correct vs incorrect trials) in the alpha frequency range from 8 to $12 \mathrm{~Hz}$. The first positive cluster was found in a time window ranging from -120 to $40 \mathrm{~ms}$ pre-(pseudo)word onset, and had a right anterior scalp distribution ( $p=0.036 ; T_{\text {sum }}=124.93, r=0.71 ;$ Fig. $\left.2 A\right)$. The second positive cluster was found in a time window ranging from 420 to $580 \mathrm{~ms}$ post-(pseudo)word onset, and had a central-left anterior distribution $\left(p=0.011 ; T_{\text {sum }}=168.95, r=0.61\right.$; Fig. $\left.2 B\right)$. Please note that the response was given at the end of the trial after the prompt occurred at $1200 \mathrm{~ms}$ post-(pseudo)word onset.

To illustrate the nature of the phase effects underlying the significant BI results, we extracted the single-participant phase angles for both positive clusters, and plotted the circular distance between mean phase angles for correct versus incorrect trials (Fig. 2A, B, bottom). For example, at electrode F6, 9 of 11 participants have a mean prestimulus phase distance $>\pi / 2$ rad $\left(90^{\circ}\right)$ leading to a consistently positive BI.

\section{Lexical-decision accuracy was not predictable from phase coherence, power, or ERP amplitude}

Accuracy of lexical decision could not be predicted based on any of the other neural measures (Fig. 3). First, no ITPC differences were observed in a nonparametric permutation test using the same time and frequency parameters as for the BI analysis (Fig. 3A; cluster closest to statistical significance with $p=0.81 ; T_{\text {sum }}=73.96, r=$ $0.68)$. Second, one cluster was observed in which absolute alpha power was higher for incorrect than for correct trials $(p=0.037$; 
A

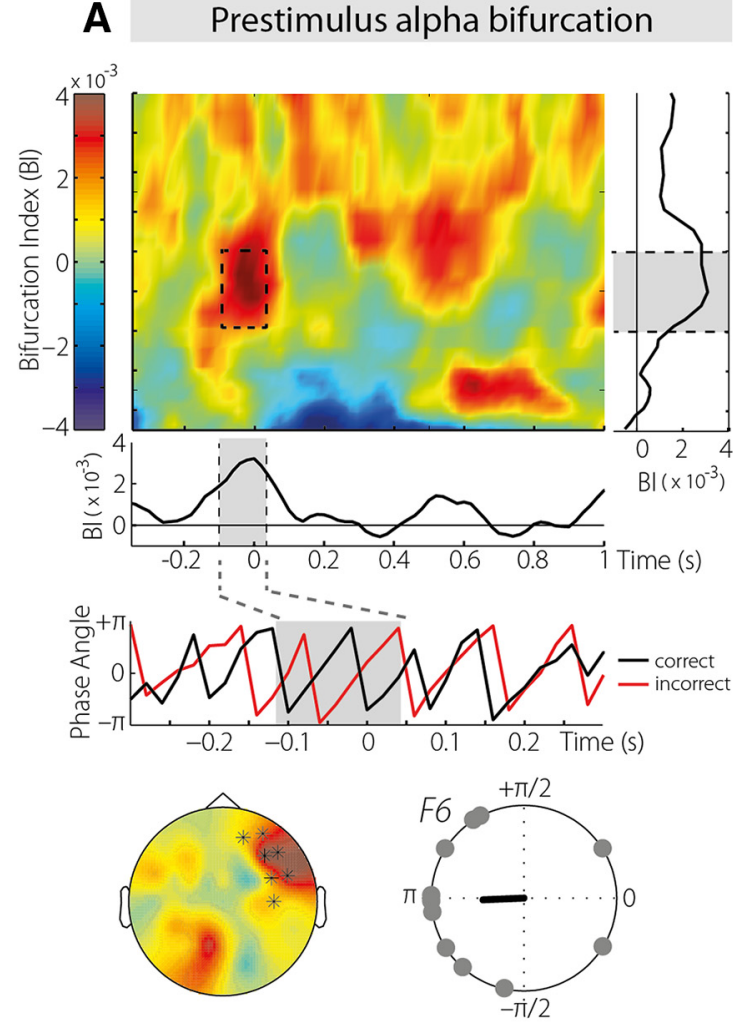

B Peristimulus alpha bifurcation
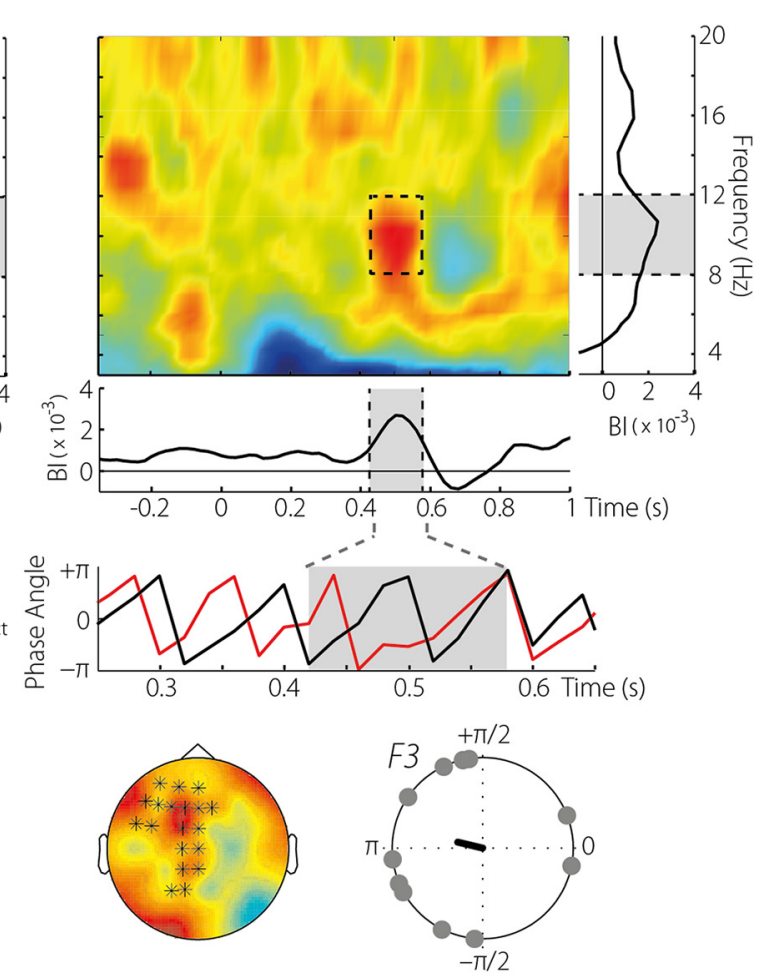

Figure 2. Results from Bl analysis. $A$, Prestimulus alpha phase bifurcation (time-frequency plots). One cluster was found in the alpha band ( $8-12 \mathrm{~Hz}$ ) with right anterior scalp distribution. Correct and incorrect trials differed in their mean phases between -120 and $40 \mathrm{~ms}[0 \mathrm{~ms}$ is (pseudo-)word onset]. Below, alpha phase extracted from and averaged over the cluster is shown in radians for correct (black) and incorrect (red) trials separately as a function of time. Phase differences (per subject) are plotted for electrode F6 along with resultant vector. $\boldsymbol{B}$, Peristimulus alpha bifurcation. Second cluster was found in the alpha band ( $8-12 \mathrm{~Hz}$ ) with left anterior central scalp distribution. Conditions differed in their mean phases from 420 to $580 \mathrm{~ms}$ [0 ms is (pseudo)word onset]. Alpha phase extracted from and averaged over the cluster is shown in radians as a function of time, for correct (black) and incorrect (red) trials separately. Phase differences (per subject) are plotted for electrode F3. Electrodes belonging to significant clusters are highlighted in topographies as asterisks.
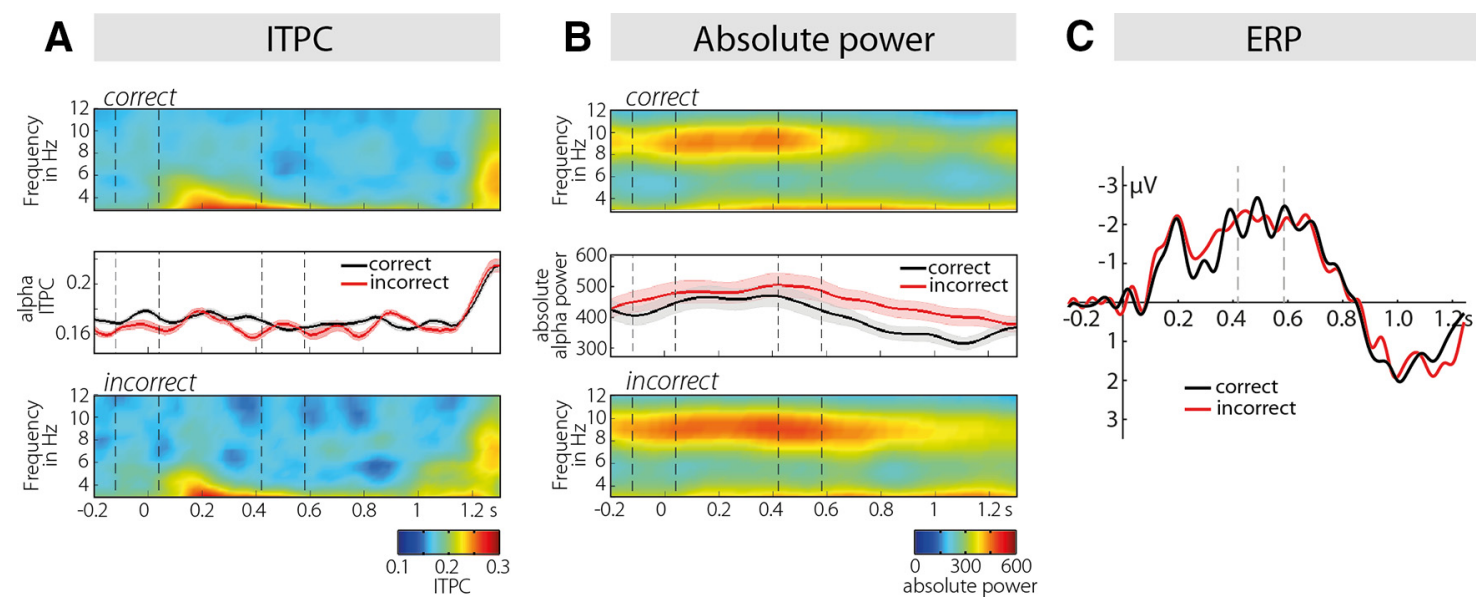

Figure 3. Time-frequency and time-domain analyses. A, ITPC shown separately for correct (top) and incorrect (bottom) trials. ITPC specifically for the alpha (8-12 Hz) band is shown in the middle, separately for correct (black) and incorrect (red) trials. No differences were observed in the alpha or theta bands. Vertically dotted lines mark the time window of the prestimulus and peristimulus alpha phase bifurcations. Shades in time-series plot (middle) depict \pm 1SEM. B, Absolute power shown for correct (top) and incorrect (bottom) trials. Conditions diverged only in a late time window after both alpha phase effects (see middle, which shows alpha-band power for correct trials in black and incorrect trials in red). C, ERPs. No effect of correct (black) versus incorrect (red) trials was found.

$\left.T_{\text {sum }}=-3619.8, r=0.60\right)$. However, the cluster comprised only lower alpha frequencies (peak at $8.3 \mathrm{~Hz}$ ) in a later poststimulus time window (peak at $0.98 \mathrm{~s}$ post-(pseudo)word onset) and over more posterior electrodes (peak at CP1; Fig. 3B). Third, evoked potentials did not show any difference for the accuracy contrast during the same time interval and over the same electrodes as the peristimulus alpha phase effect $\left(p=0.13 ; t_{(10)}=-1.65, r=0.46\right.$; Fig. $\left.3 C\right)$. In sum, these results support the notion that neural phase in the alpha frequency range was the best predictor for lexical decisions in noise.

\section{Phase effects in the theta band}

Nonparametric permutation tests of the BI against zero also revealed a negative cluster in the theta frequency range from 3 to 5 $\mathrm{Hz}$. The negative cluster ranged between 120 and $580 \mathrm{~ms}$ post- 

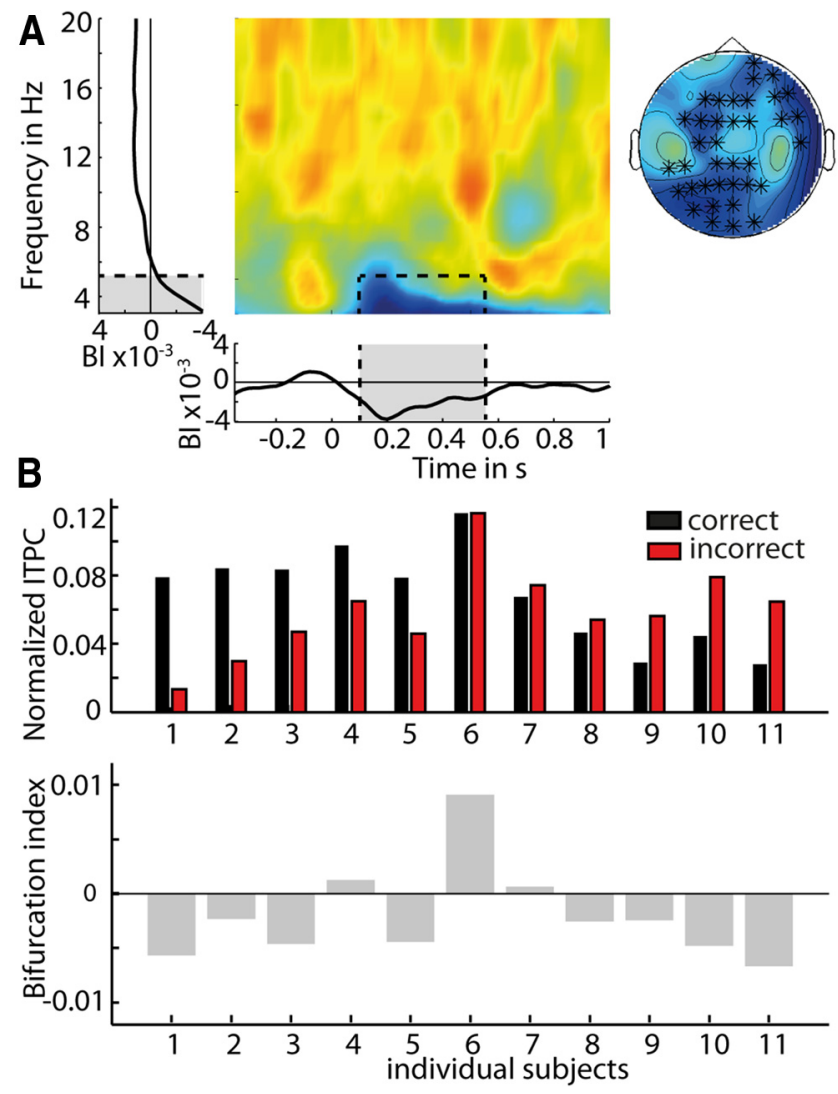

Figure 4. Theta-band phase effects are not consistent across participants. $\boldsymbol{A}$, Negative $\mathrm{BI}$ cluster. A negative cluster was found in the theta band $(3-5 \mathrm{~Hz})$ with a widespread scalp distribution in the time window ranging from 120 to $580 \mathrm{~ms}$ [0 ms is (pseudo)word onset]. $\boldsymbol{B}$, Mean BI was consistently negative despite inconsistent individual data. The bar plot (middle) shows normalized ITPC for correct (black bars) and incorrect (red bars) trials as required by the BI formula (see Materials and Methods). Normalization is obtained by subtracting the ITPC of all trials from the ITPC of correct and incorrect trials, respectively. Conditions differ in both directions across participants, leading to a consistently but misleadingly negative BI (bottom).

(pseudo)word onset, and was broadly distributed over electrodes $\left(p<0.001, T_{\text {sum }}=-1987.8, r=0.69\right.$; Fig. $\left.4 A\right)$.

Generally, a negative BI emerges in cases where neural oscillations in one condition are more phase-locked than in another condition (Busch et al., 2009). Therefore, a negative BI should be followed-up by a comparison of ITPC. In our case, it was surprising at first glance that a whole-brain cluster-based permutation test did not reveal any ITPC differences for the accuracy contrast. On closer inspection, however, the theta effect resulted from some participants showing stronger phase locking for correct than for incorrect trials and other participants showing the opposite pattern (Fig. 4B). This, somewhat misleadingly, led to a negative BI that survives statistical testing across participants.

\section{Discussion}

The current experiment examined the impact of slow neural oscillatory phase on word recognition. Going beyond previous work on neural phase effects in low-level perceptual tasks, we show that alpha $(8-12 \mathrm{~Hz})$ phase determines the accuracy of lexical decisions in perceptually uncertain situations (i.e., when stimuli are embedded in noise). The alpha phase bifurcation emerged first in a prestimulus ( $-75 \mathrm{~ms}$ pre-(pseudo)word onset) time window, but attained significance also in a peristimulus (500 ms post-(pseudo)word onset) time window.

\section{Alpha phase reflects fluctuations in the probability of attentional selection}

For near-threshold stimulation, prestimulus alpha phase has been found to determine psychophysical detection performance (Mathewson et al., 2009; Dugué et al., 2011; Neuling et al., 2012). Consistent with and extending these results, we found a prestimulus alpha phase effect for a lexical decision task in noise, whereby stimuli that were judged correctly versus incorrectly coincided with opposite prestimulus phases of the ongoing alpha oscillation, respectively. On incorrect trials, the initial phonemes of the stimulus would thus coincide with suboptimal "windows" for sensory input (Dugué et al., 2011); that is, the inhibitory phase of an ongoing alpha oscillation.

We observed the prestimulus alpha bifurcation effect over right anterior electrodes. Although the nature of this index as a first-level statistic prevents an informed interpretation of underlying neural sources (Busch et al., 2009), this location is nevertheless consistent with previous studies that have observed the recruitment of a right frontal network (for review, see Corbetta et al., 2008). Most notably, right middle frontal gyrus, frontal eye fields (Lee et al., 2014), and the right anterior insula (Eckert et al., 2009; Erb et al., 2013; Wilsch et al., 2014) have been found to be activated in particular during challenging auditory tasks. Potentially, involvement of these structures, also associated with selective attention, would have been necessary here to isolate speech from the noise background. Importantly, alpha activity has been argued to be a neural means of selecting a relevant sensory object (for more detailed discussion, see Mathewson et al., 2011; Strauß et al., 2014a). Moreover, the current alpha phase results are in line with the idea of Schroeder and Lakatos (2009) that low-frequency neural oscillatory phase correlates with the fluctuations of the probability that a stimulus is "selected" by attention. On this view, stimuli arriving in the optimal (excitatory) phase of the alpha oscillation are selected by attention and are thus more likely to be thoroughly processed and correctly judged than stimuli arriving in the suboptimal phase.

In case of word recognition, the word-initial phonemes are crucial for lexical access (Greenberg, 1999), and therefore have been emphasized in models of auditory word recognition (Taft and Forster, 1976; Marslen-Wilson, 1987). The initial phonemes are required to initiate lexical search, that is, to recruit top-down information from the mental lexicon, which is helpful to perform the lexical decision task in noise accurately. Thus, we tentatively suggest that the optimal alpha phase reflects successful attentional selection of word-initial phonemes, which led ultimately to more accurate lexical-decision performance.

\section{Alpha phase reflects decision weighting}

In the current study, we also observed an additional peristimulus alpha phase bifurcation over left frontotemporal regions. Interestingly, this peristimulus alpha phase effect occurred directly after the crucial vowel manipulation, but was not phase-locked to the onset of the vowel. (Note that a repetition of the bifurcationindex analysis time-locked to vowel onsets did not reveal any significant clusters). This favors a decision-related interpretation of the observed phase effect over a more stimulus-related interpretation. Please note, however, that the response of the lexical decision was prompted and thus was given only at the end of the trial such that reaction times could not be related to the current alpha phase effects.

The dissociation of perceptual from decisional stages and their dependence on slow neural phase is difficult in low-level detection paradigms, but has been demonstrated recently by Wyart et al. (2012) in a visual discrimination task that involved integrating visual information over $\sim 2 \mathrm{~s}$ to discriminate the mean orienta- 
tion of a series of Gabor patches. They found that the accumulation of perceptual evidence is not linear (for review, see Ratcliff and McKoon, 2008; Mulder et al., 2014; as assumed previously by a number of prominent models of decision making), but proceeds rhythmically. Moreover, integration and weighting of decisional information were also found to be coupled to low-frequency neural phase, but were critically dissociated from (earlier) accumulation of perceptual evidence.

The left anterior distribution of the later, peristimulus alpha bifurcation is compatible with the common finding of left inferior frontal gyrus involvement in visual and auditory lexical decision tasks (Fiebach et al., 2002; Xiao et al., 2005). Especially BA 45 (i.e., pars triangularis) has been suggested to receive information from inferior temporal gyrus (Heim et al., 2009) via the ventral stream (Hickok and Poeppel, 2007) presumably to support lexical selection when lexical access is difficult (Fiebach et al., 2002). Importantly, our data suggest that this selection process in left anterior cortical structures might in part be mediated via alpha-band oscillatory activity.

One remaining question concerns the relationship between prestimulus and peristimulus alpha phase in our data. We suggest that our prestimulus and peristimulus alpha phase effects reflect dissociable perceptual and decisional processes, respectively. Prestimulus and peristimulus bifurcation indices were not directly correlated (Spearman's $\rho=0.2 ; p>0.5$ ), suggesting at least partially independent mechanisms. Their independence is also supported by the observed difference in topographical distribution and would be in line with the interpretation of dissociable earlier perceptual and later decisional weighting (Wyart et al., 2012). In particular, our data are consistent with the necessity of achieving an optimal neural state not only during anticipation of a stimulus (for optimizing accumulation of perceptual evidence) but also during preparation of lexical decisions during and after the stimulus (for optimizing decisional weighting and integration).

\section{Accuracy is not predicted by other neurophysiological measures}

Strikingly, lexical decision accuracy was not predictable from other measures of neural activity, such as the amplitude of the ERP, absolute alpha power, or ITPC in the alpha band in our data (Fig. 3). That is, differences in instantaneous alpha phase seem to exhibit an independent effect on lexical decision processes and might index mechanisms that have so far not been subject to closer electrophysiological examination.

\section{Theta versus alpha phase effects on lexical decision}

Last, even though recent models of speech processing have provided good arguments to assign a crucial role to theta band oscillations (Ghitza, 2011; Gagnepain et al., 2012; Giraud and Poeppel, 2012), theta phase here was not predictive of accuracy. As a more technical aside, the multiplicative nature of the phase bifurcation index makes it insensitive to which condition is causing the negative sign of the bifurcation index. In our case, this feature could have led to the unwarranted conclusion of consistent theta-phase effects based on bifurcation statistics only. Our analysis shows that ITPC analyses are important to control for false positives when using the phase bifurcation index, specifically when the observed bifurcation index is negative.

Speculatively, the current finding (i.e., consistent predictability of response accuracy by alpha, but not by theta phase) might be due in part to the type of manipulation (short-lived vowel manipulations in isolated words) or to embedding of (pseudo) words in noise, prompting an alpha- rather than theta-driven neural processing strategy (for the functional dissociation of alpha and theta activity during word recognition, see Strauß et al., 2014b). In sum, the available evidence from this study renders alpha but not theta phase at two separate points in time and in space a good predictor of accurate lexical decisions in noise.

\section{Conclusion}

This study constitutes a first step toward characterizing neural phase signatures of higher cognitive processes, such as the ones that enable spoken word recognition in noise. Our data demonstrate that alpha phase (both before and during the presentation of word or word-like stimuli) predicts the accuracy of lexical decisions in noise. The data suggest that alpha phase acts not only to select stimuli for perceptual processing, but might also underlie rhythmic fluctuations in decisional weighting. We suggest that dependence on rhythmic fluctuations in neural excitability is encouraged in particular when perceptual evidence is limited (due for example to the presence of background noise) as is often the case in naturalistic listening conditions. Therefore, both sensory processing, as well as decision-making, proceeds coupled to ongoing internal alpha rhythms that in turn modulate performance.

\section{References}

Busch NA, VanRullen R (2010) Spontaneous EEG oscillations reveal periodic sampling of visual attention. Proc Natl Acad Sci U S A 107:1604816053. CrossRef Medline

Busch NA, Dubois J, VanRullen R (2009) The phase of ongoing EEG oscillations predicts visual perception. J Neurosci 29:7869-7876. CrossRef Medline

Corbetta M, Patel G, Shulman GL (2008) The reorienting system of the human brain: from environment to theory of mind. Neuron 58:306-324. CrossRef Medline

Cravo AM, Rohenkohl G, Wyart V, Nobre AC (2013) Temporal expectation enhances contrast sensitivity by phase entrainment of low-frequency oscillations in visual cortex. J Neurosci 33:4002-4010. CrossRef Medline

Debener S, Thorne J, Schneider TR, Viola FC (2010) Using ICA for the analysis of multi-channel EEG data. In: Simultaneous EEG and fMRI: recording, analysis, and application (Ullsperger M, Debener S, eds), pp 121-134: Oxford UP.

Ding N, Simon JZ (2013) Power and phase properties of oscillatory neural responses in the presence of background activity. J Comput Neurosci 34:337-343. CrossRef Medline

Doelling KB, Arnal LH, Ghitza O, Poeppel D (2014) Acoustic landmarks drive delta-theta oscillations to enable speech comprehension by facilitating perceptual parsing. Neuroimage 85:761-768. CrossRef Medline

Dugué L, Marque P, VanRullen R (2011) The phase of ongoing oscillations mediates the causal relation between brain excitation and visual perception. J Neurosci 31:11889-11893. CrossRef Medline

Eckert MA, Menon V, Walczak A, Ahlstrom J, Denslow S, Horwitz A, Dubno JR (2009) At the heart of the ventral attention system: the right anterior insula. Hum Brain Mapp 30:2530-2541. CrossRef Medline

Erb J, Henry MJ, Eisner F, Obleser J (2013) The brain dynamics of rapid perceptual adaptation to adverse listening conditions. J Neurosci 33: 10688-10697. CrossRef Medline

Fiebach CJ, Friederici AD, Müller K, von Cramon DY (2002) fMRI evidence for dual routes to the mental lexicon in visual word recognition. J Cogn Neurosci 14:11-23. CrossRef Medline

Gagnepain P, Henson RN, Davis MH (2012) Temporal predictive codes for spoken words in auditory cortex. Curr Biol 22:615-621. CrossRef Medline

Ghitza O (2011) Linking speech perception and neurophysiology: speech decoding guided by cascaded oscillators locked to the input rhythm. Front Psychol 2:130. CrossRef Medline

Giraud AL, Poeppel D (2012) Cortical oscillations and speech processing: emerging computational principles and operations. Nat Neurosci 15: 511-517. CrossRef Medline

Greenberg S (1999) Speaking in shorthand: a syllable-centric perspective for understanding pronunciation variation. Speech Commun 29:159-176. CrossRef

Gross J, Hoogenboom N, Thut G, Schyns P, Panzeri S, Belin P, Garrod S 
(2013) Speech rhythms and multiplexed oscillatory sensory coding in the human brain. PLoS Biol 11:e1001752. CrossRef Medline

Haegens S, Nácher V, Luna R, Romo R, Jensen O (2011) $\alpha$-Oscillations in the monkey sensorimotor network influence discrimination performance by rhythmical inhibition of neuronal spiking. Proc Natl Acad Sci U S A 108:19377-19382. CrossRef Medline

Hanslmayr S, Aslan A, Staudigl T, Klimesch W, Herrmann CS, Bäuml KH (2007) Prestimulus oscillations predict visual perception performance between and within subjects. Neuroimage 37:1465-1473. CrossRef Medline

Heim S, Eickhoff SB, Ischebeck AK, Friederici AD, Stephan KE, Amunts K (2009) Effective connectivity of the left BA 44, BA 45, and inferior temporal gyrus during lexical and phonological decisions identified with DCM. Hum Brain Mapp 30:392-402. CrossRef Medline

Henry MJ, Obleser J (2012) Frequency modulation entrains slow neural oscillations and optimizes human listening behavior. Proc Natl Acad Sci U S A 109:20095-20100. CrossRef Medline

Hickok G, Poeppel D (2007) The cortical organization of speech processing. Nat Rev Neurosci 8:393-402. CrossRef Medline

Kayser C, Ince RA, Panzeri S (2012) Analysis of slow (theta) oscillations as a potential temporal reference frame for information coding in sensory cortices. PLoS Comput Biol 8:e1002717. CrossRef Medline

Keil J, Müller N, Hartmann T, Weisz N (2014) Prestimulus beta power and phase synchrony influence the sound-induced flash illusion. Cereb Cortex 24:1278-1288. CrossRef Medline

Lachaux JP, Rodriguez E, Martinerie J, Varela FJ (1999) Measuring phase synchrony in brain signals. Hum Brain Mapp 8:194-208. CrossRef Medline

Lakatos P, Shah AS, Knuth KH, Ulbert I, Karmos G, Schroeder CE (2005) An oscillatory hierarchy controlling neuronal excitability and stimulus processing in the auditory cortex. J Neurophysiol 94:1904-1911. CrossRef Medline

Lee AK, Larson E, Maddox RK, Shinn-Cunningham BG (2014) Using neuroimaging to understand the cortical mechanisms of auditory selective attention. Hear Res 307:111-120. CrossRef Medline

Levitt H (1971) Transformed up-Down methods in psychoacoustics. J Acoust Soc Am 49:467-477. CrossRef Medline

Maris E, Oostenveld R (2007) Nonparametric statistical testing of EEG- and MEG-data. J Neurosci Methods 164:177-190. CrossRef Medline

Marslen-Wilson WD (1980) Speech understanding as a psychological process. In: Spoken language generation and understanding (Simon JC, ed), pp 39-67. Netherlands: Springer.

Marslen-Wilson WD (1987) Functional parallelism in spoken wordrecognition. Cognition 25:71-102. CrossRef Medline

Mathewson KE, Gratton G, Fabiani M, Beck DM, Ro T (2009) To see or not to see: prestimulus alpha phase predicts visual awareness. J Neurosci 29: 2725-2732. CrossRef Medline

Mathewson KE, Lleras A, Beck DM, Fabiani M, Ro T, Gratton G (2011) Pulsed out of awareness: EEG alpha oscillations represent a pulsed-inhibition of ongoing cortical processing. Front Psychol 2:99. CrossRef Medline

Mulder MJ, van Maanen L, Forstmann BU (2014) Perceptual decision neurosciences: a model-based review. Neuroscience 277:872-884. CrossRef Medline

Neuling T, Rach S, Wagner S, Wolters CH, Herrmann CS (2012) Good vibrations: oscillatory phase shapes perception. Neuroimage 63:771-778. CrossRef Medline

Ng BS, Schroeder T, Kayser C (2012) A precluding but not ensuring role of entrained low-frequency oscillations for auditory perception. J Neurosci 32:12268-12276. CrossRef Medline
Ng BS, Logothetis NK, Kayser C (2013) EEG phase patterns reflect the selectivity of neural firing. Cereb Cortex 23:389-398. CrossRef Medline

Oostenveld R, Fries P, Maris E, Schoffelen JM (2011) FieldTrip: open source software for advanced analysis of MEG, EEG, and invasive electrophysiological data. Comput Intell Neurosci 2011:156869. CrossRef Medline

Peelle JE, Davis MH (2012) Neural oscillations carry speech rhythm through to comprehension. Front Psychol 3:320. CrossRef Medline

Raettig T, Kotz SA (2008) Auditory processing of different types of pseudowords: an event-related fMRI study. Neuroimage 39:1420-1428. CrossRef Medline

Ratcliff R, McKoon G (2008) The diffusion decision model: theory and data for two-choice decision tasks. Neural Comput 20:873-922. CrossRef Medline

Rice DM, Hagstrom EC (1989) Some evidence in support of a relationship between human auditory signal-detection performance and the phase of the alpha cycle. Percept Mot Skills 69:451-457. CrossRef Medline

Romei V, Gross J, Thut G (2010) On the role of prestimulus alpha rhythms over occipito-parietal areas in visual input regulation: correlation or causation? J Neurosci 30:8692-8697. CrossRef Medline

Rosenthal R (1994) Parametric measures of effect size. In: The handbook of research synthesis (Cooper H, Hedges LV, eds), pp 231-244. New York: Russell Sage Foundation.

Rosenthal R, Rubin DB (2003) r Equivalent: a simple effect size indicator. Psychological Methods 8:492-496. CrossRef Medline

Schroeder CE, Lakatos P (2009) Low-frequency neuronal oscillations as instruments of sensory selection. Trends Neurosci 32:9-18. CrossRef Medline

Schubert R, Haufe S, Blankenburg F, Villringer A, Curio G (2009) Now you'll feel it, now you won't: EEG rhythms predict the effectiveness of perceptual masking. J Cogn Neurosci 21:2407-2419. CrossRef Medline

Spaak E, de Lange FP, Jensen O (2014) Local entrainment of alpha oscillations by visual stimuli causes cyclic modulation of perception. J Neurosci 34:3536-3544. CrossRef Medline

Strauß A, Wöstmann M, Obleser J (2014a) Cortical alpha oscillations as a tool for auditory selective inhibition. Front Hum Neurosci 8:350. CrossRef Medline

Strauß A, Kotz SA, Scharinger M, Obleser J (2014b) Alpha and theta brain oscillations index dissociable processes in spoken word recognition. Neuroimage 97:387-395. CrossRef Medline

Taft M, Forster KI (1976) Lexical storage and retrieval of polymorphemic and polysyllabic words. J Verbal Learn Verbal Behav 15:607-620. CrossRef

van Dijk H, Schoffelen JM, Oostenveld R, Jensen O (2008) Prestimulus oscillatory activity in the alpha band predicts visual discrimination ability. J Neurosci 28:1816-1823. CrossRef Medline

Varela FJ, Toro A, John ER, Schwartz EL (1981) Perceptual framing and cortical alpha rhythm. Neuropsychologia 19:675-686. CrossRef Medline

Wilsch A, Henry MJ, Herrmann B, Maess B, Obleser J (2014) Alpha oscillatory dynamics index temporal expectation benefits in working memory. Cereb Cortex. Advance online publication. Retrieved March 2, 2014. CrossRef Medline

Wyart V, de Gardelle V, Scholl J, Summerfield C (2012) Rhythmic fluctuations in evidence accumulation during decision making in the human brain. Neuron 76:847-858. CrossRef Medline

Xiao Z, Zhang JX, Wang X, Wu R, Hu X, Weng X, Tan LH (2005) Differential activity in left inferior frontal gyrus for pseudowords and real words: an event-related fMRI study on auditory lexical decision. Hum Brain Mapp 25:212-221. CrossRef Medline 